\title{
A Field Study of Pedestrians and Autonomous Vehicles
}

\author{
Samantha Reig \\ Carnegie Mellon University \\ Pittsburgh, PA, USA \\ sreig@cs.cmu.edu
}

\author{
Samadrita Das \\ Carnegie Mellon University \\ Pittsburgh, PA, USA \\ samadrid@cs.cmu.edu
}

\author{
Selena Norman \\ Carnegie Mellon University \\ Pittsburgh, PA, USA \\ stnorman@andrew.cmu.edu
}

\author{
Aaron Steinfeld \\ Carnegie Mellon University \\ Pittsburgh, PA, USA \\ steinfeld@cmu.edu
}

\author{
Cecilia G. Morales \\ Carnegie Mellon University \\ Pittsburgh, PA, USA \\ cgmorale@andrew.cmu.edu
}

\author{
Jodi Forlizzi \\ Carnegie Mellon University \\ Pittsburgh, PA, USA \\ forlizzi@cs.cmu.edu
}

\begin{abstract}
Autonomous vehicles have been in development for nearly thirty years and recently have begun to operate in real-world, uncontrolled settings. With such advances, more widespread research and evaluation of human interaction with autonomous vehicles (AV) is necessary. Here, we present an interview study of 32 pedestrians who have interacted with Uber AVs. Our findings are focused on understanding and trust of AVs, perceptions of AVs and artificial intelligence, and how the perception of a brand affects these constructs. We found an inherent relationship between favorable perceptions of technology and feelings of trust toward AVs. Trust in AVs was also influenced by a favorable interpretation of the company's brand and facilitated by knowledge about what AV technology is and how it might fit into everyday life. To our knowledge, this paper is the first to surface AV-related interview data from pedestrians in a natural, real-world setting.
\end{abstract}

\section{Author Keywords}

Autonomous vehicles; field study; trust; human-vehicle interaction.

\section{CCS Concepts}

- Human-centered computing Field studies

\section{INTRODUCTION}

Autonomous vehicles (AVs) have been in development for nearly thirty years [30] and have been operating regularly in realistic settings since 2007 [29]. Today, autonomous transport is of great interest in both research and commercial domains and has also garnered great publicity [4]. For example, in 2018, TechWorld reported that 18 companies are

Permission to make digital or hard copies of all or part of this work for personal or classroom use is granted without fee provided that copies are not made or distributed for profit or commercial advantage and that copies bear this notice and the full citation on the first page. Copyrights for components of this work owned by others than ACM must be honored. Abstracting with credit is permitted. To copy otherwise, or republish, to post on servers or to redistribute to lists, requires prior specific permission and/or a fee. Request permissions from Permissions@acm.org.

AutomotiveUI '18, September 23-25, 2018, Toronto, ON, Canada

(C) 2018 Association for Computing Machinery.

ACM ISBN 978-1-4503-5946-7/18/09\$15.00

https://doi.org/10.1145/3239060.3239064 developing AVs; these companies ranged from automobile manufacturers such as General Motors to leading technology companies such as Apple [26].

While a number of research and design techniques have been developed to understand the experience of interacting with AVs in simulated settings [36], little opportunity has been afforded to observe human interaction with actual AVs operating in real-world settings. This study is one of the first to attempt this process.

Furthermore, most of the attention in the space of human interaction with AVs has been focused on driver-vehicle interaction, which is only part of the equation. Pedestrian bystanders are a special class of interactants due to their relative lack of physical protection and lack of direct knowledge of vehicle intent $[25,36]$. To better understand how pedestrians and AVs will interact and ensure safe interactions between pedestrians and AVs, more knowledge about how pedestrian bystanders perceive AVs is needed.

In this paper, we present an interview study of pedestrian interactions with AVs. We conducted interviews with 32 pedestrians over several weeks (some during the summer, some during the winter). Here, we present findings from interviews focused on understanding and perceived trustworthiness of AVs, perceptions of AVs and artificial intelligence (AI), perceptions of the role of AVs in society, and how perception of brand affects these constructs.

In the next section, we present the related work that informed our study. We then present the study design and describe the site of the research. Following that, we present our findings, and we conclude with design implications gleaned from our study with the hope of assisting in future research and design of human-autonomous vehicle interaction.

\section{RELATED WORK}

Studies of human-autonomous vehicle interaction

On-road testing of AV behavior with participants is not very common due to the novelty of autonomous technology, logistical challenges, and the inherent risks associated with doing human studies in these contexts. Therefore, most onroad research studies have taken place in controlled settings rather than on open roads and highways. While data is being collected by Google, Uber, and other companies as part of 
their currently active field tests, findings from these proprietary experiments are rarely disclosed to the research community.

In academic settings, there have been several advances in research methods to understand the experience of AVs. One study offered a novel method to explore the interactions between AVs, cyclists, and pedestrians. The Ghost Driver method utilized a car seat costume that masked the presence of a driver, making a regular car appear to pedestrians as if it were operating autonomously [36]. Using this method, researchers could observe behavior patterns and anomalies for example, at intersections where pedestrians had to cross the street in front of an AV - and make use of those observations in the development of human-autonomous vehicle interaction. In early field studies, the majority of participants believed that the Ghost Driver vehicle was truly autonomous, which makes it a promising method for studying pedestrian-AV interaction.

The Marionette system allowed researchers to observe driver interference behavior in a simulated autonomous vehicle. In a proof-of-concept test of the system, steering wheel motion from a gaming wheel positioned in front of the participant (the "driver") was transmitted to the real steering wheel, controlled by the experimenter, who sat in the driver's seat. Using this system, participants in a study could choose to intervene and execute actions if they thought the "autonomous" vehicle was operating in an unsafe way [42]. These actions were not directly executed by the vehicle via the participant's wheel, but communicated to the experimenter, who could then execute them from the actual driver's seat. The system was found to effectively mimic transfer of control in an AV system, but in an evaluation of the system, participants stated that they did not trust the system and had trouble believing that the system really replicated a real-world AV system.

Researchers have tested driver-vehicle interactions with AVs on controlled roads to explore human factors issues specific to autonomy, most notably for hand-offs and mode selection $[6,7]$. There have also been numerous studies on precursor systems, like collision warning, driver assistance, and lane keeping systems (e.g. [5, 7, 40]). However, these types of studies typically focus on issues associated with real-time control and vehicle safety.

Collectively, these studies provide knowledge about perceptions, attitudes, and beliefs about how autonomous vehicles will operate. They also focus on initial experiences with vehicle autonomy rather than reactions to AVs after some familiarity has already developed. Their findings cannot necessarily generalize to interactions with real-world AVs, and their designs do not test perceptions of the technology over time, especially with respect to pedestrians.

\section{Studies of trust and perception of AVs}

There have been several research efforts that have studied trust and perception of AVs. This body of research generally indicates varying levels of trust for autonomous technology, but also a desire to have the ability to control it at any time, particularly in the event of error or failure. Desai and colleagues [13] presented data from a survey that queried participants about how much they would trust a car that parked autonomously for them. In the survey, $65 \%$ of respondents indicated that they would be most comfortable manually parking their own car. Respondents were also uncomfortable with the idea that an autonomous car might park next to theirs. Findings from this survey confirm other empirical studies that show that negative attitudes towards autonomous technology will negatively affect interactions with them [31].

Another study presented a survey to understand the factors that affect how people trust automation in driverless cars and medical devices [11]. Key factors included performance statistics, research on the reliability of the autonomous product or service, existence or indication of errors, and possibility of failure, among others.

Other studies explore participants' ethical judgments of autonomous vehicles. Bonnefon and colleagues [8] found that even though participants approve of autonomous vehicles that might sacrifice passengers to save others, they would prefer not to ride in these autonomous vehicles. Interestingly, the appearance of the technology also affects people's ethical judgments of it [24].

Recent work has explored how specific behaviors of AVs play into pedestrian perceptions of trustworthiness. For example, aggressive driving is likely to negatively impact pedestrian trust of AVs [20]. Similarly, AVs that have the capacity to provide explanations for their behaviors may also elicit more trust from pedestrians [16]. Communication of $\mathrm{AV}$ intent is a crucial and burgeoning research area, and one which we intend to further explore in follow-up studies.

Brand has also been shown to affect trust of autonomous vehicles [19]. One report examined nine case studies exploring measures of trust as affected by brand of medical devices and AVs. Results showed that a symbiosis exists between how trust is constructed by the public and how much the company creating the technology is trusted. In addition, suggestions were given for companies to use to increase public trust in the autonomous systems that they create.

Studies of acceptance of AVs independent of brand have suggested that public opinion can fluctuate rapidly. Sener and colleagues [39] applied a Car Technology Acceptance Model (CTAM) to a survey of adoption and trust of AVs in three Texas cities and evidenced that the psychological variables implicated in the CTAM are better predictors of perceptions about AVs than any demographic variables. Two surveys deployed by Abraham et al. [1] suggested that people were less comfortable with fully autonomous cars in 2017 than they were in 2016 and that fear of vehicle malfunction and lack foresight on the part of programmers may have increased over that time. Recently, an Uber autonomous vehicle was involved in a fatal crash; though the exact causes of the accident and the relevance of the fact that the car was autonomous have not yet been determined, it is likely that the involvement of an AV in the event will reinforce some of the apprehension about AVs. 


\section{Research on design methods for understanding human- autonomous vehicle interaction}

The discipline of design plays an important role in depicting future experiences of technology products that do not yet exist. Design and its associated research methods are critical for understanding how human interaction with AVs will take place. Design can be used to envision these futures and make them tangible to research participants and to the public at large. A design approach to envisioning future human interaction with AVs affords the opportunity to understand the current state, and then to synthesize, sketch, and prototype a preferred future state that is a desired outcome [45]. This is particularly important for technologies for which the general public's lack of familiarity limits their ability to envision the value and utility of the technology.

There are a number of design methods that allow a design and development team to prototype interaction and the social experience of a new technology product or service [12, 34]. For example, when prototyping the interior of an airplane, researchers created a chalk and chair mockup that allowed them to act out scenarios about travel and to understand what product features might be needed [10]. These methods help shrink the gap between the now and what might be.

Other methods focus on selecting particular solutions to develop from a vast solution space $[2,9,16]$. For example, the design space of social robots that recover from errors is vast, with hundreds of possible ways in which robots can behave to restore relationships with users prior to the occurrence of errors. One paper reported on the use of storyboard scenarios to quickly sample the design space to understand what people might desire before committing resources to developing the technology itself [22].

Interim prototypes of this type function as tools for discovery, as the design and development team uses them to probe the interaction between people, technology, and workflow, and to test the impact of new technologies in real world contexts [44]. They have been used in the domain of autonomous driving before: Pettersson and $\mathrm{Ju}$ [33] surveyed a number of design prototyping methods used in the domain of AVs, including enactments, contextual inquiry, scale scenarios, field experiments, video and animation prototyping, setting the stage (when a simple representation of a car serves as a stage for vehicle interaction), and Wizard-of-Oz studies.

Wizard-of-Oz studies have been conducted both in simulation and in real-world settings. The Wizard on Wheels Project at VW Germany featured a prototype that enabled varying levels of control from full driver control to full simulated automation via a second driver [38]. Using this prototype, study participants could take over full control from the "autonomous" driver. The RRADS (Real Road Autonomous Driving Simulation System) used video to simulate an autonomous driving situation, helping study participants suspend disbelief and experience what it is like to ride in an autonomous vehicle [2]. Other systems employ vehicle simulators [17] and immersive virtual reality environments [20] to provide participants the experience of interacting with "AV" technology. The Ghost Driver prototype, described earlier, helps to collect empirical data in real-world settings through the use of a car seat cover that conceals a driver, making a vehicle seem as if it were autonomous [36].

While prototyping methods have greatly advanced knowledge of human-autonomous vehicle interaction, there is some concern about the validity of the data beyond the study environments. Researchers have noted that the use of Wizard of $\mathrm{Oz}$ prototypes may not be relevant for all situations [13] and that demonstrational prototypes have high numbers of constraints and are rarely used iteratively [35]. Other researchers feel that prototypes must be high in fidelity to capture the true experiential aspects of HRI [43].

Collectively, these works are indicative of a growing research area, but their findings are limited to lab studies, Wizard of $\mathrm{Oz}$ studies, and surveys that collect data out-of-context. Design methods can be used to scaffold what we have yet to experience, giving researchers and designers insights about what people will want or will not accept. Our work builds on the knowledge from these areas of related work. With the ability to observe real-world interactions, we can create new knowledge about the relationship between humans and AVs as systems gain agency.

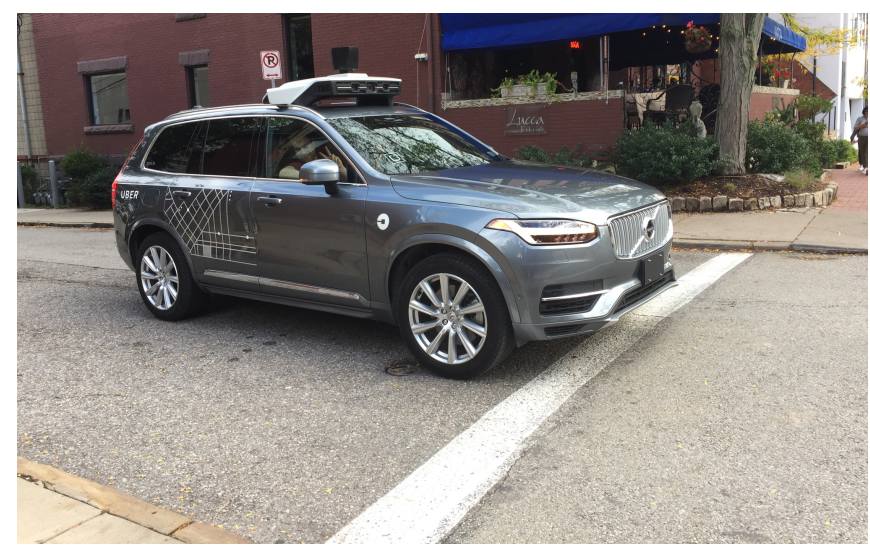

Figure 1. Image of Uber AV on the street.

\section{METHOD}

We conducted an interview-based field study of autonomous vehicles with pedestrians in nine settings in an urban location in the U.S. where autonomous Uber vehicles were routinely being tested at the time. We interviewed 32 participants over ten weeks across summer and winter seasons. This study was approved by our Institutional Review Board.

\section{Study Design}

Through interviews, we sought to answer the following research questions:

- Do pedestrians understand the operation and capabilities of AV technology in real-world settings?

- Do pedestrians trust AV technology, based on their interactions with it in real world settings?

- What are pedestrians' perceptions of AVs and AI, in general, based on their observation and experience? 
- Does perception of brand affect pedestrians' understanding, trust, or perception of AVs?

\section{Participants}

We performed intercept interviews with a total of 32 pedestrians. By chance, we recruited one participant who was a graduate student in a highly technical program. We reviewed this participant's interview responses for nontechnical content and found none. As such, we labeled this participant an outlier and did not consider data from this interview in our final analysis. This left us with a count of 31 interview participants. Of these, 14 were male, 16 were female, and 1 chose not to answer the gender question. Fifteen participants were age 40 or older, and 16 were between 18 and 40 .

After the first set of summer interviews, we determined that more data were needed and concluded that interviewing at different times of the year would give us an opportunity to learn about the potential influences of season and temperature in on pedestrians' impressions of AVs. As such, 23 of the participants were interviewed during the summer, and 8 were interviewed during the winter. All interviewees were paid $\$ 10$ for their time. Data collection finished prior to the fatal Uber AV crash.

\section{Procedure}

Interviewers waited near intersections where AVs were spotted regularly. When an AV and a pedestrian were both in the vicinity of an intersection and the pedestrian was not talking on a cell phone or seemingly in a rush, the interviewer(s) intercepted the pedestrian for an interview.

Immediately upon being approached, participants were informed that the authors were an academic research team not affiliated with any car manufacturer or developer of autonomous technology - and asked to spend a few minutes discussing their thoughts about autonomous vehicles. All participants verbally consented to being audio recorded (for transcription purposes). The interviewer then spoke to the participant for about 10 minutes on average (interviews ranged from 5 to 15 minutes long). The interview consisted of collecting demographic information, soliciting opinions about AVs and AI along either five or eight Likert-type scales, and then discussing freeform questions regarding AV failure, communication about the state of an $\mathrm{AV}$ to drivers and pedestrians, and their general knowledge and impressions of AVs (Table 1). The post-Likert portion of the interview was guided by the freeform questions, but sometimes deviated; many participants told stories of friends' experiences with AVs or explained what they believed would come of AV technology in the near future.

\section{Site of the research}

We conducted our research in an urban location in the US where autonomous passenger Uber vehicles have routinely been tested. The study began 9 months after the vehicles were introduced to the city, thereby providing time for residents to accumulate exposure to the vehicles. The terrain in the city offers a variety of road types, traffic patterns, and weather conditions, which make it a good testbed for the technology. Uber has noted that real-world testing is critical

\begin{tabular}{|c|c|c|}
\hline $\begin{array}{c}\text { Demo- } \\
\text { graphics }\end{array}$ & $\begin{array}{l}1 . \\
2 . \\
3 .\end{array}$ & $\begin{array}{l}\text { Age } \\
\text { Gender } \\
\text { Education }\end{array}$ \\
\hline $\begin{array}{l}\text { Likert- } \\
\text { type } \\
\text { ratings } \\
(1-5, \text { with } 1 \\
\text { as } \\
\text { "strongly } \\
\text { disagree" } \\
\text { and } 5 \text { as } \\
\text { "strongly } \\
\text { agree" }\end{array}$ & $\begin{array}{l}1 . \\
2 . \\
3 . \\
4 . \\
5 . \\
6 . \\
7 . \\
8 .\end{array}$ & $\begin{array}{l}\text { Autonomous driving is important } \\
\text { for me. } \\
\text { Autonomous driving is important } \\
\text { for society. } \\
\text { Autonomous vehicles are } \\
\text { trustworthy. } \\
\text { I am interested in autonomous } \\
\text { vehicles. } \\
\text { I am interested in artificial } \\
\text { intelligence. } \\
\text { Artificial intelligence is important } \\
\text { for me. } \\
\text { Artificial intelligence is important } \\
\text { for society. } \\
\text { Artificial intelligence is trustworthy. }\end{array}$ \\
\hline $\begin{array}{l}\text { Freeform } \\
\text { questions }\end{array}$ & & $\begin{array}{l}\text { Describe the traffic situation when } \\
\text { you saw the AV drive by. Did it } \\
\text { seem to be traveling smoothly? Did } \\
\text { other drivers/nearby pedestrians } \\
\text { seem to respond to the AV? Did } \\
\text { they seem to trust it? } \\
\text { Do you think Uber AVs are safe? } \\
\text { Why or why not? } \\
\text { Have you heard much about Uber } \\
\text { AVs? If so, what sources did you } \\
\text { learn information from? } \\
\text { Do you trust Uber in the way it } \\
\text { develops the technology? } \\
\text { Are you worried about AVs failing? } \\
\text { If so, name three scenarios in which } \\
\text { you think they might fail. } \\
\text { If an AV fails, how could it let a } \\
\text { pedestrian, cyclist, or driver know a } \\
\text { failure is occurring? } \\
\text { What could an Uber AV have or do } \\
\text { to make you less worried about } \\
\text { failure? } \\
\text { Do you regularly use a personal } \\
\text { assistant (such as Siri or Cortana)? } \\
\text { If so, what for? } \\
\text { Would you like for a personal } \\
\text { assistant to give you updates about } \\
\text { nearby AVs and traffic patterns? } \\
\text { Do you ride a bike regularly? If so, } \\
\text { have you encountered an Uber AV } \\
\text { while riding? If yes, describe the } \\
\text { traffic situation. } \\
\text { Do you drive regularly? } \\
\text { Have you ever ridden in an } \\
\text { autonomous vehicle? If so, describe } \\
\text { the experience. } \\
\text { What do you know about how an } \\
\text { Uber AV works? } \\
\text { What do you know about how } \\
\text { artificial intelligence works? }\end{array}$ \\
\hline
\end{tabular}

Table 1. Interview items. All participants were asked to answer the demographic questions. Likert items 6-8 and freeform questions 7,8 , and 14 were only asked winter participants due to revisions to the questionnaire in the time between the summer and winter interviews. 
to developing self-driving technology. A fleet of vehicles are augmented with sensors including radar, laser scanners, and high-resolution cameras that provide a map of the environment. Passengers may be assigned to ride in a selfdriving vehicle if they request a ride on a route where a highresolution map has been generated.

Our research team observed nine settings along routes in the city where AVs routinely run. We spent a total of 28 hours in these settings during weekdays, evenings, and weekends, approaching pedestrians for interviews when appropriate.

\section{FINDINGS}

Our findings, guided by our literature review and comprised of questionnaire results and insights drawn from freeform interview questions, clustered around five themes:

- Lack of awareness leads to mistrust and made-up explanations for the behaviors of AVs.

- Belief that AV technology is not yet mature leads to a lack of perceived trustworthiness of AVs.

- An "innocent until proven guilty" mentality is prevalent with respect to AVs and trust.

- People associate AVs with AI, and both are perceived by some to be detrimental to society.

- There are brand associations, both positive and negative, that affect the perceived trustworthiness of AVs.

Lack of knowledge and information about AVs

Participants were generally not informed about AVs. Many knew nothing more than word-of-mouth folklore, or had only a vague awareness of AVs just from seeing them traversing city streets. A clear source of information about AVs in the city was lacking, and this showed in the questionnaire results and intercept interview questions. However, the interview participants that we intercepted had clearly been observing the vehicles, making sense of them in their own ways from their driving patterns. They described the AVs' movement patterns as "moving with the flow of traffic", "lagging behind", "traveling slower than other cars or slower than the speed limit", "traveling slower than I drive", or "imitating other cars pretty well".

About half of our participants (16 out of 31) claimed to have heard little explicit information about Uber AVs: six participants said that any "information" they knew came simply from observing Uber vehicles on the street, eight expressed hearing information about AVs by word-ofmouth, and two claimed to have no information at all. The second-largest group was those who had come across information about $\mathrm{AVs}$ in the popular press or on social media (ten participants), followed by those who had learned about them from riding in a company autonomous vehicle or from a company representative (three participants) and those who claimed they had done their own reading and research (two participants). We are confident that all of the participants - even those who claimed to have heard no information - had some level of experience with AVs (at

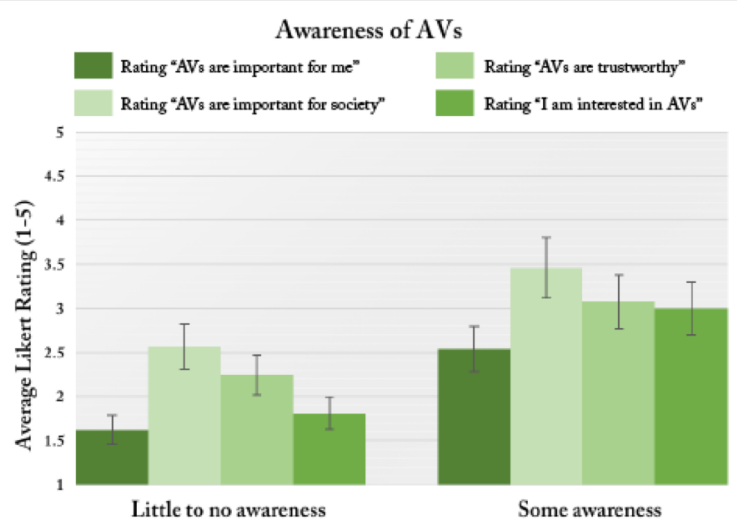

Figure 2. Average Likert ratings split by awareness of AVs (5 indicates strong agreement, bars are \pm 1 standard error).

least indirectly) because all of them confirmed having seen one pass by at the start of the interview.

Interview responses suggested that a lack of information leads primarily to two phenomena: mistrust and fear of AVs and making up explanations for behaviors in order to make sense of AVs. As an example of the former, P6 stated: "I have not heard anything about [AVs]. How do they expect me to trust in that technology if they aren't going to tell me what it is or how it works?" P22, who said he had not heard a lot about AVs, said, "I am always seeing them touching the wheel - there's something wrong with them, probably." Specific mentions of components of AV technology in freeform interview questions confirmed this. Over the 31 interviews, only two participants mentioned a map or grid and four mentioned sensors. Ten participants mentioned the role of the driver.

Many participants had their own ways of making sense of the sensors and operation of AV technology. This is in keeping with many studies in HCI and HRI that show that technology that is new or novel is open to many possible and plausible interpretations [32]. Statements about the AVs' functionality included, "If the sensor gets covered with mud, it stops working," and, "The AV is unpredictable and can fail at any time." Participants also drew their own conclusions about the safety of the technology in various situations. One participant said, "Since the driver's hands are always on the wheel of the AV, the vehicles must not be trustworthy." P32 mentioned a belief that highways are more dangerous than cities, and that if an AV were to run into a problem in a city, "the accident wouldn't be anything that I know of to be... dangerous". P24 compared the AVs' "memorizing routes in the city" to a "stunt" or "magic trick", suggesting that the advancements of AV technology were specific to the setting and would not scale to other locations. A number of creative terms were used to describe the LiDAR system that sits on top of the car's roof, including "little spinning thing", "propeller", and "whirly bird".

To better understand how participants' overall knowledge of AVs - including their familiarity with the concept of AVs, 

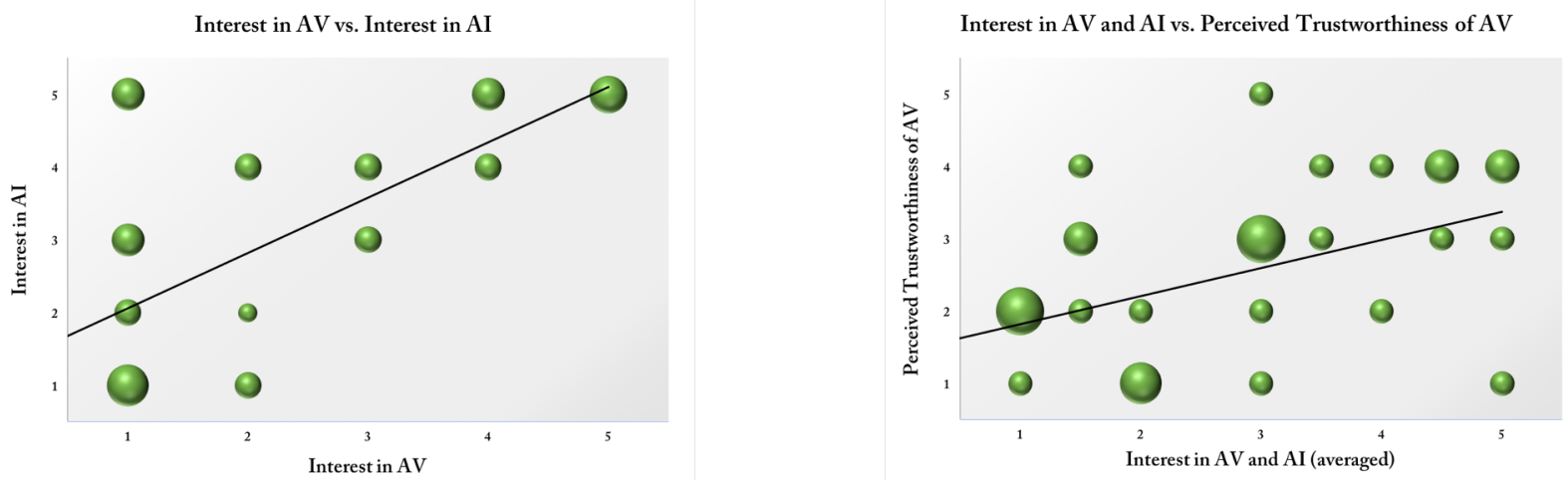

Figure 3. Comparison of interest in AI versus AV technology and comparison of interest in AV and AI technology versus trust. Bubble size represents number of participants.

their technical knowledge, their familiarity with Uber's history and current goals - influences their perceptions of AVs, we coded their interview responses to group them into three categories: Little to no awareness, Some awareness, and Informed awareness. Please see the Appendix for the coding criteria.

Based on our criteria, 17 participants fell into the Little to no awareness category, 13 had Some awareness, and 1 had Informed awareness. Because the Informed awareness category was so small, the one participant who fell into it was excluded from this analysis. We found that the influence of awareness of AVs showed up repeatedly in the Likert results. Participants who reported little knowledge of AVs tended to have lower scores for perceived importance of $\mathrm{AVs}$ to themselves, perceived importance of AVs to society, interest in AVs, and the importance of trust (Figure 2).

\section{Belief that technology is not yet mature}

Our data indicated that a number of our participants trusted an $\mathrm{AV}$ with a human driver more than an $\mathrm{AV}$ alone. We reasoned that due to the new nature of the technology, it seemed more reliable when paired with human vigilance in the form of a driver who could step in if and when the system made an error. For example, P20 stated, "I think they are safe if there is someone driving but I DON'T think they are safe if there is no one behind the wheel." P9 saw the AV stop abruptly and reasoned that the driver had taken over control of the vehicle, "I saw the AV stop abruptly when a guy cut it off. I think that was a situation where the driver in the AV intervened to stop safely." P12 stated, "I think the idea of a car driving itself is wrong - kind of like a dog driving a car."

Our data also reflected awareness of improper bystander behavior. For example, while reflecting on the behavior of a pedestrian preparing to enter an intersection, P5 noted, "That guy the Uber detected should have not been so close to the edge." These findings, along with the findings from our study, indicate that the presence of people in the operation of autonomous technology is not yet understood. The norms of a setting, typical social behaviors, and beliefs about the intelligence of the technology all play a role in how people develop trust in it [23, 37, 41].

On the other hand, many of the participants believed that once the technology is mature, it will be safer than a human driver. For example, P8 stated, "I think once they get there they will absolutely be more safe than human drivers. You can program them with the most finite detail.” P31 said, "Over time, I expect [AVs will] become more reliable", and P24 said, "I see them as babies". P26 mentioned a fear of "lack of rehearsal" and said that "unreliable tech can be dangerous"; many other participants echoed this sentiment, expressing general concerns about technology rather than fears specific to the Uber AVs that are currently on the road in the city where the interviews were conducted.

\section{AVs are "innocent until proven guilty"}

Some participants implied that with respect to their trust in AVs, they maintained an "innocent until proven guilty" mentality. Several of the participants said that they thought AVs were safe and/or trustworthy because they had not heard of any major accidents. Only one of the participants who had a generally positive outlook on AVs (P30) approached this idea of liability from the opposite direction, noting that he would need to see positive consumer reviews and statistics before believing that AVs in general are safe. Many participants were more concerned about human error than AV error: P29 said, "I'm more worried about people in cars... people are more unpredictable", and P30 said, "I can name more human error situations... than artificial intelligence [error situations]".

\section{Associations between AVs and Al and perception that they are detrimental to society}

In our study, we a found significant positive correlation between interest in AVs and interest in $\mathrm{AI}(n=31, r=0.62$, $p<.01$; Figure 3, left) with means in the low to neutral range (Interested in AVs, $m=2.4, s d=1.5$; Interested in AI, $m=$ $3.3, s d=1.6)$. In the second group of interviews, interest in $\mathrm{AVs}$ and a belief that AI is important for society were correlated $(n=8, r=0.81, p<0.05)$ (participants in the first group were not asked about their belief in the importance of AI to society). Taken together, these findings suggest that participants associate AVs and $\mathrm{AI}$ in concept.

Many participants expressed negative feelings about AVs and AIs with respect to their role in society. These feelings centered around the ideas that society is not ready for AI, that AI infringes on privacy, that it is taking away jobs, and that it cannot make moral judgments. For example, P13 stated, "I 
don't think society is ready for AVs and artificial intelligence." P21 stated, "I don't really trust autonomous technology as a whole. Everything that is man-made is going to fail... like when cell phones expire after 2-3 years. I don't know if these cars will fall into that." P19 noted, "It's common to see people not crossing the road when an AV goes by, because if people cannot stop them, what then?"

There were also comments that revealed that participants were considering the ethical implications of technology that tracks human behavior and learns from its patterns, as does AI. For example, P7 commented, "Do I think the technology is safe? No. Do I think it is all right for everything to be monitored and mapped out? No. I think it is weird and an invasion of personal space." P16 stated, "Technology is going to take away jobs and who will be displaced? What will happen to society?" Reflecting on the idea of communication between autonomous cars, other intelligent devices, and humans to indicate state and intent (which has been suggested by prior research [e.g. 16, 18, 20] and is being actively studied in the research community), P25 said that getting updates about nearby AVs would mean "another car knowing where my car is", which "could be a good thing or a bad thing". P24 held an extremely negative view of AVs because of their potential negative societal impacts and in spite of their safety, worrying that they would widen the gap between the "haves" and the "have-nots", and calling an AV a "safe car, dangerous class tool".

Participants brought up culturally relevant topics that are often present in ongoing dialogues about the pros and cons of emerging technologies. Some weighed the good against the bad: "I think there's always a wonderful path for new tech... I also worry about jobs for people who drive for delivery, long distance truckers, who knows? Where are they going to work? Will it displace them?" (P21). P31 said, "I like the convenience, but... is it a good social trend. You know, the whole gig economy thing. No benefits, erratic hours, no unionization. These are all things to be wary about." Two participants mentioned the moral dilemma in which an AV has to choose the lesser of two evils, killing either one passenger or four pedestrians $[8,24]$. In general, negative feelings about AVs and AI led to negative trust ratings ( $n=31, r=0.44, p<.05$; Figure 3 , right).

P2 had an experience during the study that made him feel positively about autonomous technology. P2 observed a pedestrian walking close to the edge of the sidewalk, and noted that the AV's sensors interpreted that the pedestrian was in the road and stopped quickly. P2 stated, "This AV was turning left, everything was fine but there was a sidewalk and someone was walking pretty close to the edge. When the AV made that turn I could see the body of the car shift as if it hit its brakes in response to the person on the sidewalk. Never seen it do that, which was pretty cool. Shows you that these things are really in the works to becoming intelligent and safe." P24 also experienced something which elicited a positive reaction; he observed a cyclist crossing the street in front of an AV, and interrupted himself with an enthusiastic, "Oh, look over there, it's waiting for that biker!"

\section{Perceived trustworthiness of AV according to brand}

Prior research on trust of AVs showed that a dual constitution of trust of autonomous technology exists, constructed of trust in the technology and trust in the innovating firm [19]. This finding was reflected in our data, where we found a mixed brand perception of Uber, which in turn affected the trust in its technology. Some felt negatively about the brand, while others cited the size and established nature of the company: P17 said, "Since Uber is a large, established company, AVs are safe;" P14 stated, "I think Uber is safe because of their engineering quality," yet P10 stated, "They are developing the cars really quickly... I feel like they are a company using short cuts and cutting corners." A few participants expressed that their trust in Uber or lack thereof stemmed from its drivers and its relationships with its employees, referring to rumors about layoffs, anecdotes about strange or unprofessional interactions with drivers, and the company's willingness to take accountability for drivers' behaviors.

Some participants called out the relationship between Uber and the city chosen for the site of our study. There was some "local pride" expressed about Uber's employees and their relationship with the city. P17 stated, "I feel like Uber could support the city more," referring to potential reciprocal agreements for jobs and data sharing. P2 referred to using locals to assist with AV development, stating, "Uber, their people are from Carnegie Mellon, right? They are smart people and I trust that everyone involved is taking this seriously." Additionally, participants referenced local cultural driving norms and how the Uber system failed to observe these local norms. Interestingly, there was only one mention of Volvo (by P26), which was the brand of cars used for the AVs during the data collection period.

\section{No effect of season or demographic variables}

Twenty-three of the interviews we discuss were conducted during the summer, and eight of them were conducted during the winter. The winter interviews were conducted against a backdrop of a variety of weather conditions, ranging from sunny "sweater weather" uncharacteristic of the season to light snow and heavy rain (which, naturally, resulted in icy roads). Responses to the Likert questions did not vary by season, and there were no noticeable tendencies in the freeform responses associated with season. In line with prior work such as [39], there were also no demographic variables that were meaningfully related to beliefs and perceptions.

\section{DISCUSSION}

We found that both the answers to the questionnaire and the freeform interview questions showed a connection between favorable perceptions of AVs and trust. Favorable perceptions of technology were also influenced by a favorable interpretation of the company's brand and from some level of knowledge about what AV technology is, how it operates, and how it might fit into daily life.

\section{Knowledge and information}

There was evidence that knowledge about AVs has an important influence on perceived importance, trust, and other factors associated with acceptance. It has been evidenced that misinformation is the biggest factor leading to mistrust of AVs [19], and our data supported this claim by showing a 
relationship between lack of awareness about AVs and lack of perceived trustworthiness of AVs. This could be easily mitigated by company communication in the form of publicity in the press and communications to the public, through messages on the company app and website, and possibly street signs and other map-based notifications on routes where AVs commonly traverse.

Our findings indicate that people associate autonomous vehicles with artificial intelligence and also that a lack of awareness about AVs is associated with less belief in the importance and trustworthiness of AVs and less interest in AVs. This aligns with the higher ratings on questions by participants who had awareness of AVs (Figure 2). These findings reinforce the importance of using design techniques to explore human-autonomous vehicle interactions. While AVs exist as a technology product, only a small portion of the participants had had direct, extended (i.e. more than a few seconds long) interactions with the technology. Paired with the general lack of knowledge about AVs, this lack of experience likely limits participants' ability to envision the value and utility of the technology.

Perceptions about AVs are frequently measured through surveys, which often indicate that people mistrust the technology. Given that AVs are only commonplace in a few cities at present, is worth noting that most survey respondents are likely to have had amounts of firsthand interaction with $\mathrm{AVs}$ equivalent to or less than the minimum level of interaction of the participants in our study. Our findings suggest that a good deal of information, which engenders trust, may come from experience. As such, large surveys meant to examine trust in AVs should ensure careful collection of data about participants' prior experiences and knowledge of AVs, both from technical and cultural perspectives. Additionally, as researchers, we should keep in mind that - as with many areas of HCI and consistent with studies of technology acceptance - opinions about AVs can vary with time spent around the technology. One-time surveys may serve to capture a snapshot of a population's perceptions at one time, whereas change-over-time studies such as [1] can more clearly evidence a trend.

\section{Feelings of trust}

Freeform interview responses also suggested that the presence of a human driver in an AV may increase feelings of trust. Humans are expected to play an important role in watchfulness and safety as AV technology matures. In reality, human monitors may be needed in AVs for an extended time until the technology is mature [27]. Even beyond that point, humans behind the wheel or otherwise in the car could serve the important role of making passengers and pedestrians feel safe around AVs.

We also found that there seemed to be an "innocent until proven guilty" mentality surrounding AVs. Many participants' comments reflected the belief that since they had not heard about any incidents in which a person was or could have been harmed by an autonomous vehicle, it was logical to continue to assume that AVs are safe. All of our data were collected before the fatal crash on March 19, 2018, involving an Uber AV in Arizona. It will be interesting to see if this incident significantly changes perceptions of AVs and if so, whether that applies to all AVs, only Uber AVs, AVs in Arizona, AVs operated by a large company, or some other subset of AVs.

\section{Communication of AV intent}

Our participants described how signaling might be useful in informing pedestrians and cyclists that an $\mathrm{AV}$ is in operation and communicating the AV's intent and current state to nearby pedestrians and other drivers. Many participants suggested loud noises such as horns and sirens as well as bright, blinking, or flashing lights. Some suggested text messages sent to dashboards and phones to signal that AVs are operating normally; others dismissed this suggestion when we offered it. A few participants suggested messages on small screens on the vehicle.

Research teams are currently exploring communication from AVs to pedestrians and other drivers $[25,28]$, which is crucially important to the experience of pedestrians in the context of AVs. Additional research has shown that the way the signal is designed can even affect people's inclinations towards, trust in, and moral judgments about the technology [24]. More research is needed to discern what the most effective AV-pedestrian failure scenario communication strategies are and how various signals of intent may influence trust and judgment of AVs.

\section{General goodwill towards AVs and Al}

Interestingly, the five Likert questions asked of all participants (see Table 1) were significantly, positively correlated and agreement among the set was reasonably strong. The set had Cronbach's $\alpha=0.84$ and stayed above 0.79 when any of the five questions were excluded. Participants in the second round of data collection were given 8 Likert questions; this set had $\alpha=0.87$ and stayed above 0.82 when excluding any of the eight questions. Across all participants, the set of questions about AVs had $\alpha$ $=0.83$, and across participants in the second round, the set of questions about AI had $\alpha=0.88$. These correlations suggest that an index of these questions may capture a broader expression of the respondent's goodwill towards AVs and AI. Formal examinations of a possible AV goodwill index have already been considered by the transportation community [13] and should continue to be explored more thoroughly, particularly with respect to pedestrians.

\section{CONCLUSION}

Autonomous vehicles have been in development for several decades and are becoming more readily viable in real-world settings. However, more widespread research, development, and evaluation of human-autonomous vehicle interaction is essential as the technology evolves and becomes road-ready. In this paper, we present an observational study of Uber AVs. We observed for a period of 28 hours over ten weeks, performing intercept interviews with 32 pedestrians (31 of which were included for analysis). We found significant correlations that indicated an inherent relationship between favorable perceptions of technology and feelings of trust in AVs. Favorable perceptions of technology were also influenced by a favorable interpretation of the company's 
brand and from clear communication to consumers about what AV technology is and how it might fit into daily life.

Our future work will explore two questions. First, we will continue to explore responses to AVs over longer time durations. Second, we will use a variety of design methods to explore the feasibility of design features that fill the need our participants expressed for communication about AV operation, intelligence, and intent.

\section{ACKNOWLEDGMENTS}

This work was funded by grants (SES-1734456 \& IIS1552256) from the National Science Foundation. This work was also supported by the Technologies for Safe and Efficient Transportation University Transportation Center (T-SET UTC), which is funded by the U.S. Department of Transportation. The authors thank Liz Carter for her detailed feedback and all of the interviewees for their participation.

\section{REFERENCES}

1. Hillary Abraham, Bryan Reimer, Bobbie D. Seppelt, and Joseph F. Coughlin. 2018. Consumer Interest in Automation: Change Over One Year. Transportation Research Board 2018 Annual Meeting.

2. Mattias Arvola and Henrik Artman. 2007. Enactments In Interaction Design: How Designers Make Sketches Behave. Artifact 1, 2, 106-119. DOI: 10.1080/17493460601117272.

3. Sonia Baltodano, Srinath Sibi, Nikolas Martelaro, Nikhil Gowda, and Wendy Ju. 2015. RRADS: Real Road Autonomous Driving Simulation. In Proceedings of the Tenth Annual ACM/IEEE International Conference on Human-Robot Interaction Extended Abstracts (HRI'15 Extended Abstracts), 283-283. DOI: 10.1145/2701973.2702099

4. Sven Beiker and Ryan Calo. 2010. Legal Aspects of Autonomous Driving. SSRN Electronic Journal. DOI: 10.2139/ssrn.2767899.

5. Klaus Bengler, Klaus Dietmayer, Berthold Farber, Markus Maurer, Christoph Stiller, and Hermann Winner. 2014. Three Decades of Driver Assistance Systems: Review and Future Perspectives. IEEE Intelligent Transportation Systems Magazine 6, 4, 6-22. DOI: 10.1109/mits.2014.2336271.

6. Myra Blanco, Jon Atwood, Holland M. Vasquez, and Sheldon M. Russell. 2016. Automated Vehicles: TakeOver Request and System Prompt Evaluation. Road Vehicle Automation 3. Springer International Publishing. 111-119.

7. Myra Blanco, Jon Atwood, Holland M. Vasquez, Tammy E, Trimble, Vicki L. Fitchett, Joshua Radlbeck, Gregory M. Fitch, Sheldon M. Russell, Charles A. Green, Brian Cullinane, and Justin F. Morgan. 2015. Human factors evaluation of level 2 and level 3 automated driving concepts. U.S. Department of Transportation National Highway Safety Administration.

8. Jean-François Bonnefon, Azim Shariff, and Iyad Rahwan. 2016. The social dilemma of autonomous vehicles. Science 352, 6293, 1573-1576. DOI: 10.1126/science.aaf2654.

9. Eva Brandt and Camilla Grunnet. 2000. Evoking the future: Drama and props in user centered design. Proceedings of Participatory Design Conference (PDC 2000), 11-20.

10. Marion Buchenau and Jane Fulton Suri. 2000. Experience prototyping. In Proceedings of the $3 \mathrm{rd}$ conference on Designing interactive systems: processes, practices, methods, and techniques (DIS '00), 424-433. DOI: $10.1145 / 347642.347802$

11. Michelle S. Carlson, Munjal Desai, Jill L. Drury, Hyangshim Kwak, Holly A. Yanco. 2013. Identifying Factors that Influence Trust in Automated Cars and Medical Diagnosis Systems. 2014 AAAI Spring Symposium Series.

12. Scott Davidoff, Min Kyung Lee, Anind K. Dey, and John Zimmerman. Rapidly Exploring Application Design Through Speed Dating. UbiComp 2007: Ubiquitous Computing. Springer-Verlag, Berlin, Heidelberg. 429-446.

13. Shuchisnigdha Deb, Lesley Strawderman, Daniel W. Carruth, Janice DuBien, Brian Smith, and Teena M. Garridson. 2017. Development and validation of a questionnaire to assess pedestrian receptivity toward fully autonomous vehicles. Transportation Research $C$, 84 (Nov. 2017), 178-195. DOI: 10.1016/j.trc.2017.08.29

14. Munjal Desai, Kristen Stubbs, Aaron Steinfeld, and Holly Yanco. 2009. Creating trustworthy robots: Lessons and inspirations from automated systems. In Proceedings of the Society for the Study of Artificial Intelligence and the Simulation of Behaviour (AISB) Convention, New Frontiers in Human-Robot Interaction.

15. Jodi Forlizzi, Thidanun Saensuksopa, Natalie Salaets, Mike Shomin, Tekin Mericli, and Guy Hoffman. Let's be honest: A controlled field study of ethical behavior in the presence of a robot. 25th IEEE International Symposium on Robot and Human Interactive Communication (RO-MAN). DOI: 10.1109/ROMAN.2016.7745206

16. Joashim Halse, Eva Brandt, Brendon Clark, and Thomas Binder. 2010. Rehearsing the Future. The Danish Design School Press.

17. Jacob Haspiel, Na Du, Jill Meyerson, Lionel P. Robert Jr., Dawn Tilbury, X. Jessie Yang, and Anuj K. Pradhan. 2018. Explanations and Expectations: Trust Building in Automated Vehicles. In Companion of the 2018 ACM/IEEE International Conference on Human-Robot Interaction (HRI '18), 119-120. DOI: 10.1145/3173386.3177057

18. Helen Hastie, Xingkun Liu, Yvan Petillot, and Pedro Patron. 2017. Talking autonomous vehicles: Automatic AUV mission analysis in natural language. OCEANS 2017,1-5.

DOI: 10.1109/OCEANSE.2017.8084617 
19. Monika Hengstler, Ellen Enkel, and Selena Duelli. 2016. Applied artificial intelligence and trust - The case of autonomous vehicles and medical assistance devices. Technological Forecasting and Social Change. 105, 105-120. DOI: 10.1016/j.techfore.2015.12.014.

20. Suresh Kumaar Jayaraman, Chandler Creech, Lionel P. Robert Jr., Dawn M. Tilbury, X. Jessie Yang, Anuj K. Pradhan, and Katherine M. Tsui. 2018. Trust in AV: An Uncertainty Reduction Model of AV-Pedestrian Interactions. HRI 2018. In Companion of the 2018 ACM/IEEE International Conference on Human-Robot Interaction (HRI '18), 133-134. DOI: 10.1145/3173386.3177073

21. David J. LeBlanc, M. Palmer, Jeremy Salinger, Richard K. Deering, and M. Shul'man. 1999. Development and validation of functional definitions and evaluation procedures for collision warning/avoidance systems. Final Report DOT HS, 808, 964.

22. Min Kyung Lee, Sara Kiesler, Jodi Forlizzi, Siddhartha Srinivasa, and Paul Rybski. 2010. Gracefully mitigating breakdowns in robotic services. 5th ACM/IEEE International Conference on Human-Robot Interaction (HRI '10), 203-210. DOI: 10.1109/HRI/2010.5453195

23. Alexandru Litoiu, Daniel Ullman, Jason Kim, and Brian Scassellati. 2015. Evidence that Robots Trigger a Cheating Detector in Humans. In Proceedings of the Tenth Annual ACM/IEEE International Conference on Human-Robot Interaction (HRI '15), 165-172. DOI: 10.1145/2696454.2696456

24. Bertram F. Malle, Matthias Scheutz, Jodi Forlizzi, John Voiklis. 2016. Which robot am I thinking about? The impact of action and appearance on people's evaluations of a moral robot. $201611^{\text {th }}$ ACM/IEEE International Conference on Human-Robot Interaction (HRI '16), 125-132

25. Aarian Marshall. 2017. Want to Teach Self-Driving Vehicles to Talk? Pretend You're a Car Seat. Wired. https://www.wired.com/story/man-dresses-carseatresearch-self-driving/, accessed April, 2018.

26. Christina Mercer and Tom Macaulay. 2018. What Companies Are Making Driverless Cars? Techworld. http://www.techworld.com/picture-gallery/data/companies-working-ondriverless-cars-3641537/, accessed April, 2018.

27. Rachel Metz. 2017. Otto Cofounder Says AI Will Be Widely Available in Trucks Within 10 Years. MIT Technology review.

https:/www.technologyreview.com/s/603919/ottocofounder-says-aiwill-be-widely-available-in-truckswithin-10-years/, accessed July, 2017.

28. Nicole Mirnig, Nicole Perterer, Gerald Stollnberger, and Manfred Tscheligi. 2017. Three Strategies for Autonomous Car-to-Pedestrian Communication: A Survival Guide. Proceedings of the Companion of the 2017 ACM/IEEE International Conference on HumanRobot Interaction (HRI '17), 209-210. DOI: $10.1145 / 3029798.3038402$
29. Michael Montemerlo et al. 2008. Junior: The Stanford entry in the Urban Challenge. Journal of Field Robotics. 25, 9, 569-597. DOI: 10.1002/rob.20258.

30. No Hands Across America. https://www.cs.cmu.edu/ tjochem/nhaa/nhaa_home_pa ge.html, accessed July 2018.

31. Tatsuya Nomura, Takayuki Kanda, Tomohiro Suzuki, and Kensuke Kato. 2008. Prediction of Human Behavior in Human-Robot Interaction Using Psychological Scales for Anxiety and Negative Attitudes Toward Robots. IEEE Transactions on Robotics. 24, 2, 442-451. DOI: 10.1109/tro.2007.914004.

32. Wanda J. Orlikowski. 1996. Improvising Organizational Transformation Over Time: A Situated Change Perspective. Information Systems Research . 7, 1, 63-92. DOI: 10.1287 /isre.7.1.63.

33. Ingrid Pettersson and Wendy Ju. 2017. Design Techniques for Exploring Automotive Interaction in the Drive towards Automation. Proceedings of the 2017 Conference on Designing Interactive Systems (DIS '17), 147-160. DOI: 10.1145/3064663.3064666

34. Ingrid Pettersson and I.C. MariAnne Karlsson. 2014. Setting the stage for autonomous cars: a pilot study of future autonomous driving experiences. IET Intelligent Transport Systems. 9, 7 (Sep. 2014), 694-701. DOI: 10.1049/iet-its.2014.0168.

35. Laurel D. Riek. 2012. Wizard of Oz Studies in HRI: A Systematic Review and New Reporting Guidelines. Journal of Human-Robot Interaction, 119-136. DOI: 10.5898/jhri.1.1.riek.

36. Dirk Rothenbücher, Jamy Li, David Sirkin, Brian Mok, and Wendy Ju. 2016. Ghost driver: A field study investigating the interaction between pedestrians and driverless vehicles. 25th IEEE International Symposium on Robot and Human Interactive Communication (RO$M A N)$. DOI: 10.1109/ROMAN.2016.7745210

37. Maha Salem, Gabriella Lakatos, Farshid Amirabdollahian, and Kerstin Dautenhahn. 2015. Would You Trust a (Faulty) Robot? Proceedings of the Tenth Annual ACM/IEEE International Conference on Human-Robot Interaction (HRI '15). DOI: $10.1145 / 2696497$

38. G. Schmidt, M. Kiss, E. Babbel, and A. Galla. 2008. The Wizard on Wheels: Rapid Prototyping and User Testing of Future Driver Assistance Using Wizard of $\mathrm{Oz}$ Technique in a Vehicle. Proceedings of the FISITA 2008 World Automotive Congress, Munich F2008-02-001

39. Ipek Nese Sener, Johanna Zmud, Thomas Williams. 2018. Measures of Baseline Intent to Use Automated Vehicles: A Case Study of Texas Cities. Transportation Research Board 2018 Annual Meeting.

40. Aaron Steinfeld and Han-Shue Tan. 2000. Development of a Driver Assist Interface for Snowplows Using Iterative Design. Transportation Human Factors 2, 3, 247-264.

41. Leila Takayama, Wendy Ju, and Clifford Nass. 2008. Beyond dirty, dangerous and dull: what everyday people 
think robots should do. Proceedings of the 3rd international conference on Human robot interaction (HRI '08), 25-32. DOI: 10.1145/1349822.1349827

42. Peter Wang, Srinath Sibi, Brian Mok, and Wendy Ju. 2017. Marionette. Proceedings of the 2017 ACM/IEEE International Conference on Human-Robot Interaction (HRI '17), 243-243. DOI: 10.1145/2909824.3020256

43. A. Weiss et al. 2009. User experience evaluation with a Wizard of Oz approach: Technical and methodological considerations. 2009 9th IEEERAS International Conference on Humanoid Robots.

44. David Woods and Klaus Christoffersen. 2001. Balancing Practice Centered Research and Design. American Psychological Association (APA).

45. John Zimmerman and Jodi Forlizzi. 2014. Research Through Design in HCI. Ways of Knowing in HCI. Springer New York. 167-18 


\section{APPENDIX}

Coding scheme for Awareness of $A V S$

If all of the responses below suggest Little to no awareness, the participant is classified as Little to no awareness. If any responses suggest Some awareness, the participant is classified as Some awareness. If any responses suggest Informed awareness, the participant is classified as Informed awareness.

\begin{tabular}{|c|c|c|c|c|}
\hline & $\begin{array}{l}\text { Question (paraphrased for } \\
\text { anonymity) }\end{array}$ & Little to no awareness & Some awareness & Informed awareness \\
\hline \multirow[b]{3}{*}{$\begin{array}{l}\text { Free Response } \\
\text { Questions - original } \\
\text { (P1-P32) }\end{array}$} & $\begin{array}{l}\text { Have you heard much publicly } \\
\text { about the AVs that are out on the } \\
\text { street? If so, what sources did you } \\
\text { learn information from? }\end{array}$ & $\begin{array}{l}\text { No knowledge beyond just knowing they're } \\
\text { around/that they exist } \\
\text { Example responses: } \\
\text { No } \\
\text { Have not heard much } \\
\text { Just from the internet }\end{array}$ & $\begin{array}{l}\text { Knowledge or interest beyond the fact of } \\
\text { their existence } \\
\text { Example responses: } \\
\text { I seek out articles on this } \\
\text { Misunderstandings about AVs in the city } \\
\text { Example responses: } \\
\text { I thought all of that company's cars in } \\
\text { this city were autonomous }\end{array}$ & $\begin{array}{l}\text { Knowledge of specifics } \\
\text { Mentions of the car company in the news } \\
\text { Personal experiences with AVs* } \\
\text { Example responses: } \\
\text { My friend works on autonomous car } \\
\text { research } \\
\text { I am a robotics student } \\
\text { (*if supported by accurate descriptions) }\end{array}$ \\
\hline & $\begin{array}{l}\text { Are you worried about AVs } \\
\text { failing? If so, name three scenarios } \\
\text { where you think they might fail. }\end{array}$ & \multirow[t]{2}{*}{$\begin{array}{l}\text { Expression of general worry, but no } \\
\text { specific scenarios }\end{array}$} & \multirow[t]{2}{*}{$\begin{array}{l}\text { Yes }+[\text { specific scenarios] } \\
\text { Use of relevant terms }\end{array}$} & \multirow[t]{2}{*}{$\begin{array}{l}\text { No/yes, because [technical } \\
\text { explanation of lack of worry] } \\
\text { Use of technical terms }\end{array}$} \\
\hline & $\begin{array}{l}\text { If an AV fails, how could it let a } \\
\text { pedestrian, cyclist or driver know a } \\
\text { failure is occurring? }\end{array}$ & & & \\
\hline \multirow[b]{3}{*}{$\begin{array}{l}\text { Free Response } \\
\text { Questions - new } \\
\text { (only P24-P32) }\end{array}$} & $\begin{array}{l}\text { Have you ever ridden in an } \\
\text { autonomous vehicle? }\end{array}$ & No & $\begin{array}{l}\text { Yes }+ \text { [somewhat accurate } \\
\text { elaboration] } \\
\text { No, but [friend/relative/colleague has] } \\
+ \text { [somewhat accurate elaboration] }\end{array}$ & $\begin{array}{l}\text { Yes }+ \text { [accurate elaboration] } \\
\text { No, but [friend/relative/colleague has] } \\
+ \text { [accurate elaboration] }\end{array}$ \\
\hline & $\begin{array}{l}\text { What do you know about how an } \\
\text { AV works? }\end{array}$ & \multirow{2}{*}{$\begin{array}{l}\text { Very inaccurate or illogical explanations } \\
\text { No technical terms } \\
\text { Claim no knowledge } \\
\text { "Explanations" that don't go beyond } \\
\text { observation } \\
\text { Example responses: } \\
\text { I know nothing about this } \\
\text { No idea } \\
\text { I know there's a person in the car } \\
\text { I have heard of artificial intelligence }\end{array}$} & \multirow{2}{*}{$\begin{array}{l}\text { Sensible explanations } \\
\text { Vague/somewhat inaccurate but still } \\
\text { logical explanations } \\
\text { Misuse of technical terms } \\
\text { Example responses: } \\
\text { The spinning LiDAR detects obstacles } \\
\text { AVs run on a grid } \\
\text { They're mapping right now }\end{array}$} & \multirow[t]{2}{*}{$\begin{array}{l}\text { Accurate explanations } \\
\text { Accurate use of technical terms }\end{array}$} \\
\hline & $\begin{array}{l}\text { What do you know about how } \\
\text { artificial intelligence works? }\end{array}$ & & & \\
\hline Extra & $\begin{array}{l}\text { (Misc. responses to other questions, } \\
\text { content related to awareness) }\end{array}$ & $\begin{array}{l}\text { Reveals fundamental misunderstanding } \\
\text { about AVs } \\
\text { Example: } \\
\text { All of that company's cars that are on the } \\
\text { road are autonomous }\end{array}$ & $\begin{array}{l}\text { Reveals more knowledge/experience } \\
\text { than expressed in response to } \\
\text { questions above } \\
\text { Example: } \\
\text { I am a mechanic }\end{array}$ & $\begin{array}{l}\text { Reveals more knowledge/experience } \\
\text { than expressed in response to } \\
\text { questions above } \\
\text { Example: } \\
\text { I am a researcher in a computer } \\
\text { science department }\end{array}$ \\
\hline
\end{tabular}

